\title{
The persistent vegetative state: time for caution?
}

\author{
Jeremy Brown
}

University College and Middlesex School of Medicine, London, UK.

As a philosophical discipline, medical ethics finds itself somewhat distant from its more established relatives in the schools of philosophy; while they may enjoy a traditional detachment from the concrete reality of everyday life, medical ethics is firmly embedded in the clinical questions which demanded its creation. Hence medical ethicists must have an intimate understanding of clinical realities to appreciate fully the difficult cases which demand their attention. These complexities are well illustrated by several studies which collected data to provide, inter alia, the basis for the ethical treatment of that group of patients who had sustained hypoxic-ischaemic brain damage and remained in what has come to be known as the persistent vegetative state. ${ }^{1-3}$ Levy attempted to produce an algorithm that would '... spare families the emotional and financial burden of prolonged care of patients with a hopeless prognosis' as well as concentrate limited resources on those most likely to gain useful and significant benefit.

These are goals of which few clinicians would disapprove. However, the results of these studies have recently been questioned by disturbing case reports which seem to undermine not only the accuracy of proposed algorithms for predicting outcome, but also our understanding of the pathology underlying the persistent vegetative state (PVS). The first was reported in July 1989 in the United States of America by Steinbock and involved an 86 year old lady who had been diagnosed as being in a PVS for 5 months following a major intercranial haemorrhage. On the basis of this, a court granted her family's request to remove the feeding tube keeping her alive. A few days after the hearing, she '... regained consciousness, took small amounts of food by mouth, and engaged . . . in conversation'. ${ }^{4}$ The report of the case fails to provide the precise clinical conditions of the case, concentrating instead on its legal and ethical implications. However, it alerts clinicians to the dangers inherent in clinical prognoses. The recent

Correspondence: Jeremy Brown, B.Sc., M.B., B.S., 3 Harrowes Meade, Edgware, Middlesex HA8 8RR, UK. Received: 30 March 1990 report by Falk in the Journal provides greater clinical detail, and is therefore more worrying. ${ }^{5}$ It details the full physical and intellectual recovery of a patient 30 weeks after hypoxic-ischaemic brain damage in whom the original prognosis was considered to be hopeless.

Given the actual outcome of the case, it is necessary to consider what may have happened had the patient been treated in accordance with some of the more recent declarations on care and management of the PVS patient. The American Academy of Neurology noted that in all postmortem studies carried out on PVS patients, cerebral damage was found to such a degree that the capacity for consciousness or the experience of pain and suffering was inconceivable. The Academy argued that there is no ethical difference between withdrawing and withholding treatment, nor any difference between providing nutrition or hydration and other forms of life-sustaining therapy. In view of all this, the Academy adopted a permissive position by allowing hydration and nutrition to be discontinued in PVS patients. ${ }^{6,7}$ While some have gone further, arguing that the PVS patient should be redefined as dead, ${ }^{8-10}$ others have been more conservative in their approach. Brody distinguishes between withdrawal of medical treatment and withdrawal of life sustaining food and fluids, arguing that what is at issue is the nature of the process which causes death. "If the cause of death is the underlying disease process itself, then allowing the patient to die from that process (assuming that appropriate consent had been given) is acceptable. However, causing death by removing essential nutrition is not allowing the disease process to run its 'natural' course, and is therefore ethically unacceptable.

Although, according to most accepted definitions, PVS patients are not defined as dead, the management of such patients remains controversial. What now needs to be assessed is how far reports of recovery from PVS demand a change in the treatment of these patients. Clearly such cases are rare, but the fact that they exist cannot be ignored. After all, clinical science should follow the same tests of falsifiability that are demanded in other scientific disciplines. If it only takes one black 
swan to disprove the thesis that all swans are white, it need only take one recovery to invalidate the thesis that all patients in a PVS with poor prognostic features will fail to achieve a reasonable and significant improvement in their lives as persons and not merely as bodies. The clinician must balance the demands of health care planners and his concern for the patient and his family to provide an honest prognosis on the basis of past experience, as well as the possibility, no matter how remote, that a prognosis may prove to be inaccurate. Isolated reports may provide unrealistic hopes for improvement, but Falk believes that without the optimism and involvement of his patient's family, it is unlikely the patient would have survived at all. Moreover, the recovery commented on by Steinbock also followed '. . . apparently as the result of aggressive efforts of the nursing staff to stimulate

\section{References}

1. Bates, D., Caronna, J.J. Cartlidge, N.E. et al. A prospective study of non-traumatic coma: Methods and results in 310 patients. Ann Neurol 1977, 2: 211-220.

2. Levy, D.E., Knill-Jones, R.P. \& Plum, F. The vegetative state and its prognosis following non-traumatic coma. Ann NY Acad Sci 1978, 315: 293-306.

3. Levy, D.E., Carrona, J.J., Singer, B.H. et al. Predicting outcome from hypoxic-ischaemic coma. JAMA 1985, 253: $1420-1426$.

4. Steinbock, B. Recovery from persistent vegetative state? The case of Carrie Coons. Hastings Center Report 1989, 19: 14-15.

5. Falk, F.H. Physical and intellectual recovery following prolonged hypoxic coma. Postgrad Med J 1990, 66: 384-386. and feed ... the patient. Perhaps then the balance has tipped again in favour of those who would wish to provide basic nutritional requirements to all PVS patients, at least preventing them from dying from anything other than a consequence of the state itself. Falk's suggestion for an international registry of unexpected coma recoveries to be maintained should be acted upon, for the diagnostic criteria and algorithmic predictive procedure for patients in a PVS may need refining. Until then, a more cautious approach should be adopted before removing life sustaining nutrition.

\section{Acknowledgement}

I am grateful to Dr Gerald Stern for stimulating some of the ideas in the paper.

6. Anon. Position of the American Academy of Neurology on certain aspects of the care and management of the persistent vegetative state patient. Neurology 1989, 39: 125-126.

7. Rachels, J. The End of Life. Oxford University Press, Oxford, 1987, pp. 100- 105.

8. Veatch, R. The definition of death; ethical, philosophical and policy confusion. Ann N Y Acad Sci 1978, 315: 307.

9. Smith, D.R. Legal recognition of neocortical death. Cornell Law Review 1983, 71: 850-862.

10. Gervais, K.G. Redefining Death. Yale University Press, New Haven, 1986.

11. Brody, B.A. Ethical questions raised by the persistent vegetative patient. Hastings Center Report 1988, 18: 33-37. 